\title{
Robust reconstruction from chopped and nodded images
}

\author{
F. Lenzen ${ }^{1}$, O. Scherzer ${ }^{1}$, and S. Schindler ${ }^{2}$ \\ 1 Institute of Computer Science, University of Innsbruck, Technikerstraße 21a, 6020 Innsbruck, Austria \\ e-mail: Frank.Lenzen@uibk . ac . at \\ 2 Institute for Astrophysics, University of Innsbruck, Technikerstraße 25, 6020 Innsbruck, Austria
}

Received 10 May 2005 / Accepted 30 July 2005

\section{ABSTRACT}

A well-known technique to reduce the influence of thermal and background noise in ground-based infrared imaging is chopping and nodding, where four different signals of the same object are recorded and from which the object is reconstructed numerically. Since noise in the data can severely affect the reconstruction, regularization algorithms have to be implemented. In this paper we propose to combine iterative reconstruction algorithms with robust statistical methods. Moreover, we study the use of multiple chopped data sets with different chopping amplitudes and the appropriate numerical reconstruction algorithm. Numerical simulations show the robustness of the proposed methods in dealing with noisy data.

Key words. methods: data analysis - techniques: image processing - infrared: general

\section{Introduction}

Data collected in infrared ground-based astronomy are affected by atmospheric and telescopic thermal background noise. A common approach for noise reduction is chopping and nodding (Emerson 1994; Robberto et al. 2005). In chopping the secondary mirror of the telescope is moved (cf. Bertero et al. 2003a) and signals are recorded for two different tilt angles. We denote the positions before and after tilting by $A$ and $B=A+h$, and the recorded signals by $S_{1}$ and $S_{2}$, respectively. Afterwards, in a nodding procedure the telescope is moved to the position $C=A-h$ and the signal $S_{3}$ is recorded. Then a second chopping procedure is applied and a signal $\tilde{S}_{1}$ is recorded at position $A$.

In the literature the vector $h$ is referred to as the chopping amplitude. The data visualized after chopping and nodding is

$f=S_{1}-S_{2}-S_{3}+\tilde{S}_{1}$

and the image $u$ to be reconstructed satisfies

$2 u()-.u(.-h)-u(.+h)=f($.$) .$

When observing a point-like and isolated object the chopping amplitude can be chosen in such way that at positions $B$ and $C$ only empty sky is recorded, in which case the numerical reconstruction algorithm is not required. However, in practice the objects observed are often extended or other objects are nearby. In these cases the reconstruction step is mandatory.

Reconstruction from chopped and nodded data was first discussed in Beckers (1994), where a Fourier-based reconstruction was suggested.
In Bertero et al. (1999, 2000, 2003a,b), iterative reconstruction algorithms were investigated. Recently Chan et al. (2003) proposed reconstruction algorithms based on wavelet decomposition.

Experiments have shown that noise can severely affect the reconstruction process (see Kaeufl 1995). In this paper we investigate two different approaches for robust reconstruction in the presence of a significant amount of noise.

The outline of this paper is as follows: in Sect. 2 we derive the model of chopping and nodding and review the reconstruction algorithms introduced by Bertero et al. (2003a,b).

In this paper we use iterative regularization techniques as well and thus Bertero's work is the most relevant to compare with. However for the sake of noise robustness we combine iterative regularization techniques with robust filtering methods from statistics (cf. Sect. 3). Moreover, we study the combined reconstruction from multiple chopped data with different chopping amplitudes and the effect on the quality of the reconstruction. Finally, in Sect. 4 we present some numerical experiments.

\section{Problem description and basic reconstruction methods}

\subsection{Continuous problem}

The problem description follows Bertero et al. (2003a,b), but differs in the way that we take into account two-dimensional chopping amplitudes. In Bertero et al. (2003a,b) the data are 
preprocessed by appropriate rotations so that the chopping amplitude is along the principal axis of the data.

We denote by $u: \mathbb{R}^{2} \rightarrow \mathbb{R}$ the brightness intensity distribution in the sky, which we assume to be non-negative.

Let $\Omega=\left[0, l_{x}\right] \times\left[0, l_{y}\right] \subset \mathbb{R}^{2}$ be a section of the sky under investigation and let $h=\left(h_{x}, h_{y}\right) \neq 0, h_{x} \geq 0, h_{y} \geq 0$ be the chopping amplitude. We define the operator

$$
\begin{aligned}
& I_{h}: L^{2}\left(\Omega_{h}\right) \rightarrow L^{2}(\Omega) \\
& u \rightarrow I_{h}(u)(x, y):=2 u(x, y)-u\left(x+h_{x}, y+h_{y}\right) \\
& \quad-u\left(x-h_{x}, y-h_{y}\right),
\end{aligned}
$$

where $Y:=L^{2}(\Omega)$ and $X:=L^{2}\left(\Omega_{h}\right)$ are the spaces of square integrable functions on $\Omega$ and

$$
\begin{aligned}
\Omega_{h}:=\Omega \cup\{(x, y) \mid & \left(x+h_{x}, y+h_{y}\right) \in \Omega \text { or } \\
& \left.\left(x-h_{x}, y-h_{y}\right) \in \Omega\right\},
\end{aligned}
$$

respectively.

The problem of reconstruction from chopped data $f(x, y)$ : $\Omega \rightarrow \mathbb{R}$ can be written as an operator equation

$$
I_{h}(u)=f
$$

A solution of Eq. (3) is also a minimizer of the functional

$$
u \rightarrow\left\|I_{h}(u)-f\right\|^{2}
$$

and therefore satisfies the corresponding optimality condition

$I_{h}^{*}\left(I_{h} u-f\right)=0$

$I_{h}^{*}$ is the adjoint operator to $I_{h}$, which satisfies

$\int_{\Omega_{h}} I_{h}(u) v=\int_{\Omega} u I_{h}^{*}(v)$ for all $u \in L^{2}\left(\Omega_{h}\right), v \in L^{2}(\Omega)$.

Since $I_{h}^{*}$ is injective, for any injective operator $J: X \rightarrow Y$, Eqs. (3), (4) and

$J\left(I_{h} u-f\right)=0$

have the same solutions. Basic numerical methods for solving Eq. (5) are based on fixed point iterations:

$u^{i+1}:=u^{i}-\tau J\left(I_{h} u^{i}-f\right), \quad u^{0}:=0$,

where $\tau$ is a relaxation parameter. For $J=I_{h}^{*}$ the fixed point iteration is commonly referred to as Landweber iteration

$u^{i+1}:=u^{i}-\tau I_{h}^{*}\left(I_{h} u^{i}-f\right), \quad u^{0}:=0$.

In Bertero et al. (2003a), Eq. (6) has been implemented with an operator $J$ that extends $u$ periodically to $\Omega_{h}$.

Here, as in Bertero et al. (2003b), we use the operator

$J(u)(x):= \begin{cases}u(x) & \text { if } x \in \Omega \\ 0 & \text { else }\end{cases}$

which extends a function $u \in L^{2}(\Omega)$ to zero in $\Omega_{h} \backslash \Omega$.

\subsection{Discretization}

The domain $\Omega$ is discretized by a quadratic grid with nodes $\left(x_{i}, y_{j}\right) \in \Omega, i=1 \ldots N, j=1 \ldots M$ and cell length 1 . The nodes coincide with the sampling points of $u$ and $f$, i.e., $\boldsymbol{u}:=$ $\left(u_{i, j}\right)=\left(u\left(x_{i}, y_{i}\right)\right)$ and $f:=\left(f_{i, j}\right)$ are the sampling data.

With $\boldsymbol{u}$ a bilinear interpolating function is associated. Assuming $u \equiv 0$ on $\Omega_{h} \backslash \Omega$ the resulting discretized system of Eq. (3) is

$A_{\mathrm{h}} \boldsymbol{u}=\boldsymbol{f}$,

where $A_{\mathrm{h}}$ is an $N M \times N M$ matrix. Details on the structure of matrix $A_{\mathrm{h}}$ and properties of its eigenvalues can be found in Bertero et al. (2003b). Note that matrix $A_{\mathrm{h}}$ is symmetric and positive definite and thus Eq. (8) has a unique solution.

Since the null-space of $I_{h}$ is not trivial, the choice of the extension of $u$ into $\Omega_{h} \backslash \Omega$ enforces a particular solution of $I_{h}$ to be calculated.

\subsection{Review on reconstruction methods}

Three different methods have been proposed in the literature for reconstruction from chopped and nodded data: Fourierbased reconstruction method (cf. Beckers 1994; Bertero et al. 2003a), iterative reconstructions (cf. Bertero et al. 2003a), and a wavelet based approach (cf. Chan et al. 2003). These methods are reviewed below:

1. For applying the Fourier-based reconstruction (cf. Bertero et al. 2003a) it is assumed that $h$ is in the vertical direction and the solution of Eq. (3) is periodic across $\partial \Omega$ in the chopping direction. In this case the image can be reconstructed for each column separately, the discretized linear system of $I_{h}$ becomes $\tilde{A}_{h} u=f$ with a circulant matrix $\tilde{A}_{h}$ and Fourier techniques can be used for an efficient numerical solution: applying the discrete Fourier transform to the linear system, it becomes

$\mathcal{F}\left(\tilde{A}_{h}\right) \mathcal{F} u=\mathcal{F} f$

where $\mathcal{F}\left(\tilde{A}_{h}\right)=D:=\operatorname{diag}\left(d_{1}, \ldots d_{M}\right)$ is diagonal, and thus can be solved efficiently provided the diagonal matrix has full rank.

2. The constrained Landweber iteration (Bertero et al. 2003a, referred to as method (A)) reads as follows:

$$
\begin{aligned}
& u^{(0)}=0 \\
& \text { for } n=1 \ldots N: \\
& \qquad u^{(n)}=P_{+}\left[u^{(n-1)}+\tau A_{\mathrm{h}}^{T}\left(f-A_{\mathrm{h}} u^{(n-1)}\right)\right]
\end{aligned}
$$

where $P_{+}$is the projection operator onto the set of nonnegative vectors (each negative entry is set to zero), and $\tau$ is a positive relaxation parameter.

The projected Lavrentiev iteration (method (B)) is defined by

$$
\begin{aligned}
& \begin{array}{l}
u^{(0)}=0 \\
\text { for } n=1 \ldots N: \\
\qquad u^{(n)}=P_{+}\left[u^{(n-1)}+\tau\left(f-A_{\mathrm{h}} u^{(n-1)}\right)\right] .
\end{array}
\end{aligned}
$$


Both iterative methods work reasonably efficiently if the chopped and nodded data are only distorted by a small amount of noise. In this case, the results are qualitatively comparable to those obtained with the Fourier methods. However, these methods suffer from robustness problems with respect to high noise distortions.

3. Finally we review a wavelet approach proposed by Chan et al. (2003). Here chopping is interpreted as high-pass filtering. Supplementing this filter by a low-pass and a subsequent high-pass filtering a tight frame wavelet system for a multi-resolution analysis is obtained. The Landweber method is then combined in a multi resolution framework and a wavelet thresholding is applied (cf. Donoho \& Johnstone 1994). Wavelet thresholding is used to denoise each iterate. Moreover, a post-processing step to remove artifacts in feature-less areas is proposed.

\section{Robust reconstructions}

\subsection{Modifications of the iterative methods}

Simulations with noisy data show that the methods described above have a tendency to introduce artificial structures as a result of the noise (cf. Sect. 4.2).

We propose to combine iterative reconstruction methods with a median filtering technique in each iteration step. Median filtering (cf. e.g. Pestman 1998) is the method of choice, since it removes artificial structures (cf. Soille 2003) appearing in each iteration step. Moreover, the median can be implemented very efficiently.

The median filter is defined as follows:

For an odd number of values $\left\{v_{1} \ldots v_{2 n+1}\right\}$ in ascending order, the median is $v_{n}$. In median filtering each value $u_{i_{0}, j_{0}}$ is replaced by the median value of surrounding values $u_{i, j}$ with indices $i, j$ in a neighborhood of $i_{0}, j_{0}$. We take the neighborhood $(i, j)$ satisfying $\left|i-i_{0}\right| \leq s$ and $\left|j-j_{0}\right| \leq s$ with $s=1,2,3$. The median filter can be implemented very efficiently (cf. Soille 2003; Sonka et al. 1999).

We investigate the following variant of method (A) defined by

$u^{(0)}=0$

for $n=1 \ldots N$ :

$$
\begin{aligned}
& \text { 1. } u^{(n)}=P_{+}\left(u^{(n-1)}+\tau A_{\mathrm{h}}^{T}\left(f-A_{\mathrm{h}} u^{(n-1)}\right)\right) \\
& \text { 2. Apply the median filter to } u^{(n)} \text {. }
\end{aligned}
$$

and the variant of method (B) is defined accordingly.

This strategy of combination of an iterative method with additional filtering after each iteration is analogous to the strategy of the wavelet based approach in Chan et al. (2003), where wavelet thresholding is used for additional filtering after each iteration of the Landweber method.

\subsection{Reconstruction based on the conjugate gradient method}

Since $A_{\mathrm{h}}$ is symmetric and positive-definite, it can be solved with the conjugate gradient (cg) method (cf. Hanke-Bourgeois 2002), which has faster convergence properties than the
Landweber and Lavrentiev methods. However, the fast convergence also makes the method more sensitive to noise, which we overcome again by applying after one iteration of the cg-method an additional median filtering step. The modified cg-method reads as follows:

$u^{(0)}=0$

for $n=1 \ldots N$ :
1. Apply one step of the cg-method with initial vector $u^{(n-1)}$
2 . Denoting the result by $\tilde{u}^{(n)}$, we replace $\tilde{u}^{(n)}$ by $P_{+} \tilde{u}^{(n)}$ to meet the constraint of non-negativity.
3. We apply the median filter to $\tilde{u}^{(n)}$ to achieve iterate $u^{(n)}$.

The constraint of non-negativity is necessary to avoid artifacts in the reconstruction.

\subsection{Multiple chopped data sets}

For an improvement of the quality of the reconstruction Beckers (1994) and Bertero et al. (2000) proposed using multiple chopped and nodded data sets. The reconstruction is performed on each data set independently and the results are combined in a post-processing step calculating the pointwise mean or the median.

In Bertero et al. (2000) this strategy is used to avoid two kinds of artifacts, first, ghosts from bright sources, and second regions where faint structures are superimposed by negative counterparts of bright sources and consequently the reconstructed image artificially becomes zero.

In the following we take advantage of multiple data sets for a robust reconstruction in the presence of noise.

Let $h^{k}, k=1 \ldots K$ denote a set of chopping amplitudes and denote by $f^{k}$ the corresponding sampled data sets.

A reconstruction $u$ is a solution of the system

$A_{\mathrm{h}^{k}} u=f^{k} k=1, \ldots, K$.

We solve this system with a blocked Landweber-Kacmarcz method (for more details on such methods cf. Kaltenbacher et al. 2005):

Set $u^{(0)}=0$

for $n=1 \ldots N$ : (iteration index)

1. For each $k=1, \ldots, K$ perform

two $\mathrm{cg}$ iterations starting from $\left(u^{(n-1)}\right)$.

The solution is denoted by $u_{k}^{(n)}$.

2 . Calculate the median $u^{(n)}$ from

of $u_{1}^{(n)}, \ldots, u_{K}^{(n)}$ for noise removal.

Note that the median is calculated separately in each sampling point for the stack of images: That is in step 2 of the above algorithm we set

$\left(u^{(n)}\right)_{i, j}:=\operatorname{median}\left\{\left(u_{1}\right)_{i, j}, \ldots,\left(u_{K}\right)_{i, j}\right\}$

for each $i, j$.

We also tested an alternative combination, namely to first detect outliers in $\left\{\left(u_{1}\right)_{i, j}, \ldots,\left(u_{K}\right)_{i, j}\right\}$ via the standard deviation and then calculating the mean ignoring these outliers, which gives fairly similar results as when using the median. 


\section{Numerical experiments}

\subsection{Simulation of the noise process}

Realistic noisy test data used for our numerical experiments require detailed knowledge of the noise process. There are two different sources of noise, which we have to take into account, background emission and thermal (dark) noise which is to be reduced with chopping and nodding, and noise occurring during the data recording process, which is Poisson noise (due to photon counting errors of CCD detector) and Gaussian readout noise.

We assume that the noise affects each of the four recorded signals independently. Let $\eta(u(x, y))$ denote the random variable, then the recorded signal reads as follows

$$
\begin{aligned}
\tilde{f}(x, y):= & 2(u(x, y))+\eta(u(x, y))+\eta(u(x, y)) \\
& -u\left(x+h_{x}, y+h_{y}\right)-\eta\left(u\left(x+h_{x}, y+h_{y}\right)\right) \\
& -u\left(x-h_{x}, y-h_{y}\right)-\eta\left(u\left(x-h_{x}, y-h_{y}\right)\right) .
\end{aligned}
$$

We write $\tilde{f}(x, y):=f(x, y)+\tilde{\eta}(x, y)$ resp. $\tilde{f}_{i, j}:=f_{i, j}+\tilde{\eta}_{i, j}$, where $\tilde{\eta}(x, y)$

$\tilde{\eta}(x, y)=\eta(u(x, y))+\eta(u(x, y))$

$-\eta\left(u\left(x+h_{h}, y+h_{y}\right)\right)-\eta\left(u\left(x-h_{x}, y-h_{y}\right)\right)$.

According to our considerations, the random variable $\eta(u)$ is the sum of a Gaussian white random $\eta_{1}=N\left(0, \sigma_{1}\right), \sigma_{1} \geq 0$ (the background emission and read out noise) and a Poisson noise $\eta_{2}(u): \eta(u):=\eta_{1}(u)+\eta_{2}(u)$.

We use test images with intensities scaled to the range of $[0,1]$. These intensities are related to the number of photons counted by the CCD detector. An intensity $u$ corresponds to the number of $N \cdot u$ photon counts with some unknown factor $N>0$. The Poisson distribution of the photon counts can be approximated by a Gaussian distribution with standard deviation $\sqrt{N \cdot u}$.

Scaling this distribution to the range $[0,1]$ the intensities are Gaussian distributed with standard deviation $\sqrt{u / N}$. Therefore, we can approximate $\eta_{2}$ with a Gaussian distribution with standard deviation $\sqrt{\sigma_{2} u}$ and $\sigma_{2}=\frac{1}{N}$.

\subsection{Behavior of the "classical" methods}

In the following we demonstrate that artificial structures appear in the reconstruction with "classical" methods from the noise.

Here we concentrate on the case of chopping amplitudes that are small with respect to the image size.

In the case of a horizontal chopping amplitude being an integer multiple of the pixel size, the condition number of the matrix $A_{\mathrm{h}}$ is of the order of the ratio of number of sampling points and the chopping amplitude (cf. Bertero et al. 2003b). Thus the problem becomes ill-conditioned for small chopping amplitudes and low-frequency eigenvectors are affected by noise in the data resulting producing the artificial structures (cf. Bertero et al. 2003b).

Figure 1 shows an artificial test image (left) and corresponding chopped and nodded data (right) with a chopping amplitude $h=(5,3)$ which is distorted by two Gaussian noise
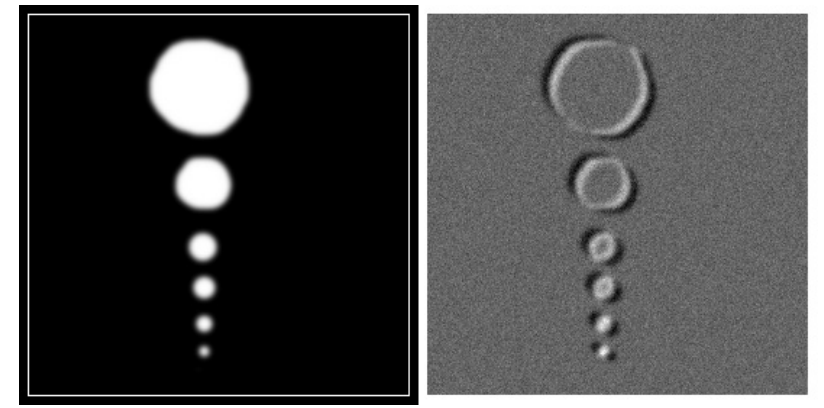

Fig. 1. Left: test image. The white frame marks the domain $\Omega$, where the chopped data are recorded; right: Corresponding chopped data with chopping amplitude $h=(5,3)$ including noise with variances $\sigma_{1}=0.05$ and $\sigma_{2}=0.0001$.

processes with parameters $\sigma_{1}=0.05$ and $\sigma_{2}=0.0001$. The signal-to-noise-ratio is $25.4752^{1}$.

Figure 2, top left and top right, shows the reconstruction from these test data applying method (B) with $\tau=0.1$ for $h=(5,3)$ after 10 and 100 iterations, respectively. We applied the same method to test data with a chopping amplitude $h=(5.5,3.3)$ not matching the grid spacing. Reconstructions after 10 resp. 100 steps are shown in Fig. 2, bottom left and right.

The computation times ${ }^{2}$ of method B were $0.05 \mathrm{~s}$ for $h=$ $(5,3)$ and 10 steps, $0.2 \mathrm{~s}$ for $h=(5.5,3.3)$ and $10 \mathrm{steps}, 0.5 \mathrm{~s}$ for $h=(5,3)$ and 100 steps and $1.9 \mathrm{~s}$ for $h=(5.5,3.3)$ and 100 steps.

Since the chopped and nodded data $f$ provide information at the objects' edges, their reconstruction is satisfactory at an early stage of the iteration, whereas the objects' interior is reconstructed at a later stage. Therefore, larger objects require a larger number of iterations to be reconstructed satisfactory.

When the discretization points are aligned with the chopped discretization points the reconstruction is more efficient. If the points are not aligned, then the numerical reconstruction is smoother; moreover, the iterative algorithms are more slowly convergent, i.e. a larger number of iterations is needed to reconstruct the interiors of the objects, which in turn leads to an amplification of noise and appearance of artificial structures. Method (A), the cg-method, and the Fourier method show similar convergence properties. Applying the cg-based method to noisy data shows that the residual $\left\|A_{\mathrm{h}} u-f\right\|$ decreases in the beginning but after some iterations it starts oscillating or even increases. This suggests stopping the iteration of the cg-solver when the residual reaches its first local minimum.

Since Eq. (3) is linear we have $I_{h}\left(u_{f}+u_{\eta}\right)=f+\eta$, where $u_{\eta}$ are the data reconstructed from noise and $u_{f}$ is the reconstruction from noise-free data.

\footnotetext{
${ }^{1}$ Let $u: \Omega \rightarrow \mathbb{R}$ be a signal with minimum $u_{\min }$ and maximum $u_{\max }$, $\tilde{u}: \Omega \rightarrow \mathbb{R}$ a distorted signal and $\sigma$ the standard deviation of $u-\tilde{u}$, then the signal-to-noise-ratio is defined by $S N R:=20 \log _{10}\left(\frac{u_{\max }-u_{\min }}{\sigma}\right)$.

${ }^{2}$ Computed on an AMD 64 FX 3500+, computations times have been averaged over several runs.
} 

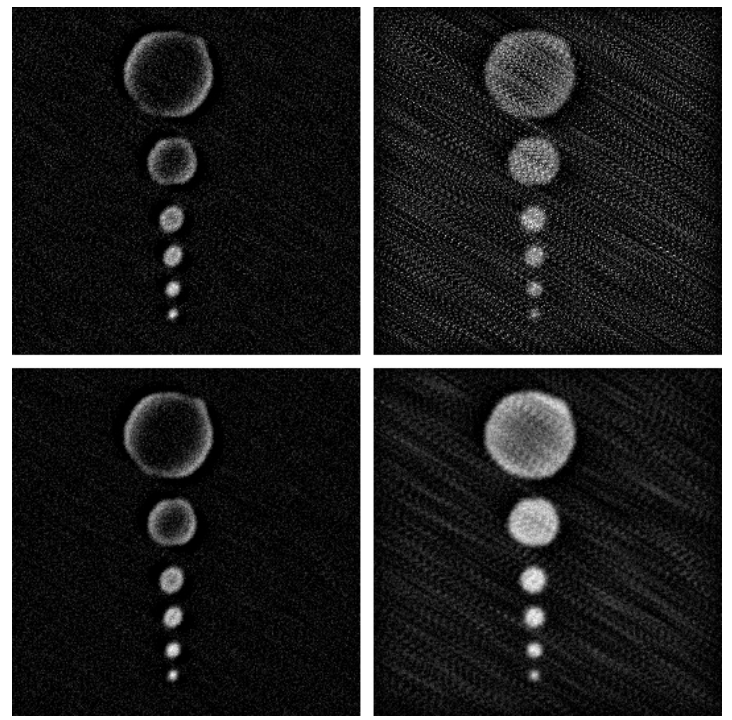

Fig. 2. Top left: reconstruction from the noisy data given in Fig. 1, right, with method (B) after 10 iterations and $\tau=0.1$. Top right: reconstruction with method (B) after 100 iterations and $\tau=0.1$. Bottom left: reconstruction with $h=(5.5,3.3)$ using method (B) after 10 iterations and $\tau=0.1$. Bottom right: reconstruction from the same data using Method (B) after 100 iterations and $\tau=0.1$.

For illustration of the noise amplification behavior of this equation, we assume that the data $f \equiv 0$ are disturbed at one grid point:

$\eta_{i, j}=\left\{\begin{array}{l}1 \text { if } i=i_{0} \text { and } j=j_{0} \\ 0 \text { else }\end{array}\right.$

for a fixed $i_{0} \in\{1 \ldots N\}, j_{0} \in\{1 \ldots M\}$.

First, let us consider the Fourier-based reconstruction from this particular $\eta$ for horizontal chopping amplitudes. In order to handle small eigenvalues of matrix $D$, we use a slightly modified method replacing matrix $D$ by

$D_{\varepsilon}^{\dagger}:=\operatorname{diag}\left(d_{1}^{\dagger} \ldots d_{N}^{\dagger}\right)$

where $\varepsilon>0$ is a small parameter and

$d_{i}^{\dagger}=\left\{\begin{array}{cc}1 / d_{i} & \text { if }\left|d_{i}\right|>\varepsilon \\ \varepsilon & \text { else }\end{array}, \quad i=1 \ldots N\right.$.

Figure 3 shows a horizontal cut through point $\left(i_{0}, j_{0}\right)$ of the function reconstructed from $\eta$ for $h=(5,0)$ and $\varepsilon=0.1$ resp. $\varepsilon=1$.

Similar artificial structures can be observed in the reconstruction from iterative methods. Figure 4 shows the reconstruction from data $f$ for $h=(5,0)$ (top) and $h=(5.5,0)$ (bottom).

In the later case, as the unknowns of $u$ are far more coupled the reconstruction is smooth and thus more complicated to handle numerically.

\subsection{Results of the modified methods}

In this section we present numerical results of the modified methods applied on noisy data.

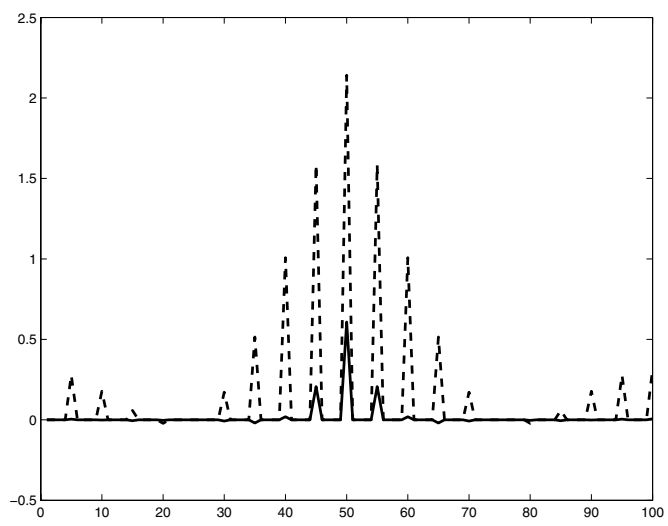

Fig. 3. Horizontal cut of the image reconstructed with the Fourier method with $D_{0.1}$ (solid line) and $D_{1}$ (dashed line). The data are $f_{i, j}:=\delta_{i_{0} i} \delta_{j_{0} j}$ for afixed $i_{0} \in\{1 \ldots N\}, j_{0} \in\{1 \ldots M\}$ and the chopping amplitude is $h=(5,0)$.
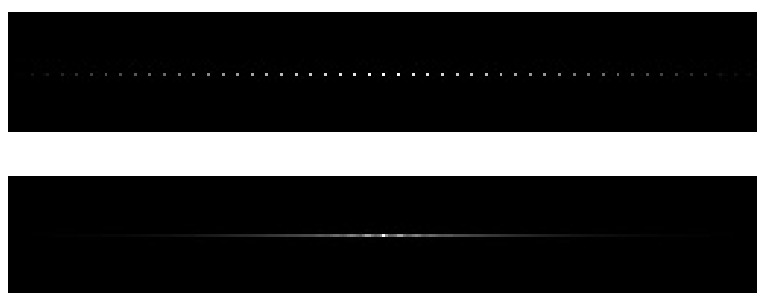

Fig. 4. Reconstruction from data $f_{i j}=\left\{1\right.$ if $i=i_{0}$ and $j=j_{0}, 0$ else $\}$, $i_{0}$ and $j_{0}$ fixed, using the cg-based method for $h=(5,0)($ top $)$ and $h=(5.5,0)($ bottom $)$.

First we used the Fourier method with inverse $D_{\varepsilon}$ to reconstruct our first test image ${ }^{3}$.

However, numerical experiments show that a small $\varepsilon$ is needed for a good reconstruction of noise-free data. Since the eigenvalues of the inverse tend to infinity for $\varepsilon \rightarrow 0$, artificial structures due to noise are significant for small $\varepsilon$. For noisy data, a compromise between good reconstruction and little noise amplification is not possible. The results are not satisfactory, so they are not presented here.

Secondly, we consider reconstructions from the cg-based method and method (B). Results from method (A), which in general are more blurred, are not presented here.

Figure 5 left, shows the reconstruction from noisy data with chopping amplitude $h=(5,3)$ with method (B) combined with median filtering (filter size $s=1$ ). Figure 5 right, shows the reconstruction using the cg-method with median filter ( $s=3$ resp. $s=1$ in the last step).

The computation times were $3.3 \mathrm{~s}$ for method (B) and $4.4 \mathrm{~s}$ for the cg-method.

In both reconstructions artificial structures arising from noise are removed by the median filtering (cf. Fig. 2).

Further experiments have been performed with the test image shown in Fig. 6, which contains an object with a wide halo extending across the boundary of the chosen domain.

Figure 6 (left) shows the signal $u$ on $\Omega_{h}$, the part in which the chopped data are provided is marked by the white rectangle.

\footnotetext{
3 For implementation the FFTW-C-library (Frigo \& Johnson 1998, resp. www. fttw.org) was used.
} 

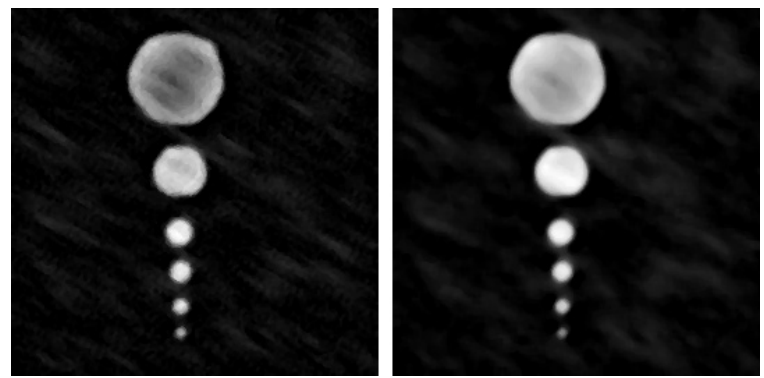

Fig. 5. Left: reconstruction from the data in Fig. 1, right, with chopping amplitude $h=(5,3)$ using method (B) with $\tau=0.1$ and 100 iterations combined with median filtering (filter size $s=1$ ). Right: reconstruction after 30 iterations of the cg-based method combined with median filtering (filter size $s=3$ resp. $s=1$ in the last iteration).
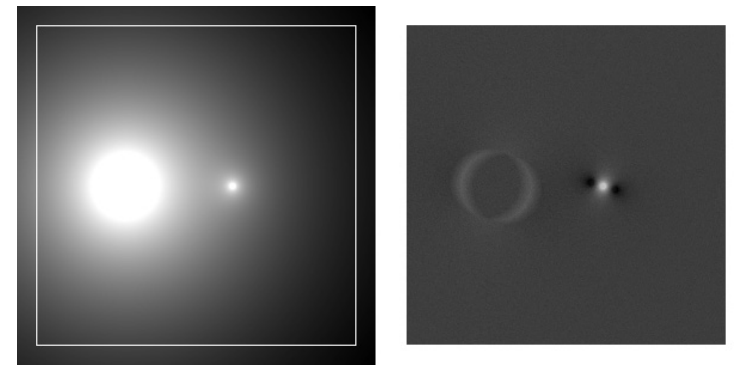

Fig. 6. Left: the second test image. Right: the corresponding noisy chopped and nodded image for $h=(10,3), \sigma_{1}=0.002$ and $\sigma_{2}=$ 0.00001 .

We produced a multiple chopped data set with 5 different chopping amplitudes $(10,3),(0,7),(10,10),(7,14)$ and $(15,0)$. The chopped data for $h=(10,3)$ on $\Omega$ is shown in Fig. 6 (right).

We added noise of variance $\sigma_{1}=0.001$ and $\sigma_{2}=0.00001$. Note that for this test image the chopped data set contains very weak structures. The signal-to-noise-ratio is 40.0494. We compare different strategies for reconstruction from noisy data. First we calculated the results of the cg-method with 40 iterations for each single data set, applying the median filter after each iteration. For a fair comparison we depict the data set showing the best results, which is that for $h=(7,14)$. Figure 7 (top left) shows the result of the cg-based method without median filtering. Undesirable noise enhancement occurs. Figure 7 (top right) shows the reconstruction with the cg-method where in each iteration step a median filtering is performed ( $s=3$ resp. $s=1$ in the last step).

Figure 7 (bottom left) shows the combined result of independent reconstructions from the multiple data set, applying 40 iterations of the cg-method without median filtering on each set and finally calculating the median in each data point. By this post-processing the artificial structures are weakened.

Figure 7 (bottom right) shows the reconstruction combining the different results after each cycle of cg-iterations by calculating the pointwise median.

The computation times were about $0.9 \mathrm{~s}$ for the cg-method without median filtering and about $5.5 \mathrm{~s}$ with median filtering. The reconstruction with multiple chopped data sets took about $4.7 \mathrm{~s}$ with independent reconstruction and finally averaging and $5 \mathrm{~s}$ with calculating the median after each iteration.
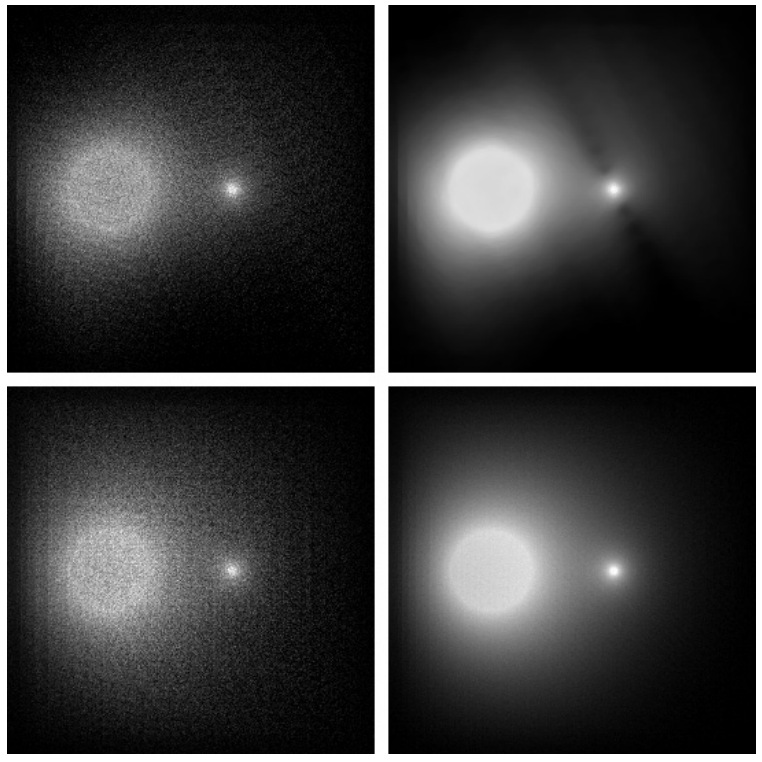

Fig. 7. Top left: reconstruction with the cg-method after 40 iterations (without median filtering). The chopping amplitude is $h=(7,14)$. Top right: 40 iterations with the cg-method combined with median filtering with filter size $s=3$ ( $s=1$ in the last iteration). Note that for comparison with the method of multiple chopped data sets with amplitudes $h=(10,3),(0,7),(10,10),(7,14)$ and $(15,0)$ we depicted $h=(7,14)$ showing best results by independent reconstruction. Bottom left: combined image using the multiple chopped data calculating the pointwise median after independent reconstruction. Bottom right: reconstruction using multiple data sets and combining the results after each iteration using the pointwise median.

The third test image (Fig. 8) is an observation of the planetary nebula Menzel III (the ant nebula). The intensities stored in floating point precision show a large difference of intensities between the nebula and the central star. To visualize the data, the intensities were logarithmically scaled to gray values by applying the function $S(x)=\frac{\log (x /(m \cdot \max )+0.1)-\log (0.1)}{\log (1.1)-\log (0.1)}$, where $x \geq 0$, max is the maximal intensity in the image and $m$ is a scaling parameter, set to $m=0.01$ for the results presented here.

We simulated the chopping and nodding procedure including artificial noise of variance $\sigma_{1}=5 \times 10^{-6}$ and $\sigma_{2}=10^{-6}$. We have chosen this noise variance corresponding to the weak intensities of the nebula in the range about $10^{-4}$. We used small chopping amplitude of up to 14 pixels for testing. Note that when applying large chopping amplitudes the original image can be reconstructed by a few iteration steps. Then the amplification of the noise is negligible. The corresponding chopped data for $h=(14,10)$ is given in Fig. 8 (right). The signal-tonoise ratio is 42.9788 . Figure 9 (top left) shows the result of method (B) after 100 iterations where at each iteration median filtering with $s=1$ has been used. Figure 9 (top right) shows the result of the cg-based method after 20 iterations in combination with median filtering. In both cases some blurring effect caused by the median filter can be recognized. Finally we used data obtained with chopping amplitudes $(14,10),(14,0)$, $(14,14),(10,0)$ and $(10,14)$. Figure 9 (bottom left) shows the reconstruction using method (B) on each data set and combining the results after each iteration step using the pointwise 

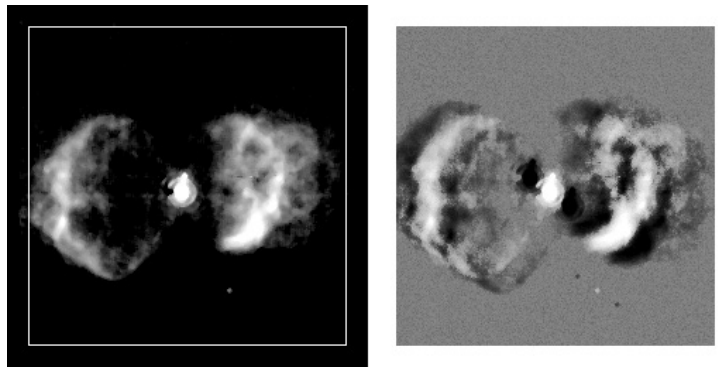

Fig. 8. Left: third test image, planetary nebula Menzel III, Right: artificial chopped data for $h=(14,10)$ including noise $\sigma_{1}=5 \times 10^{-6}$ and $\sigma_{2}=10^{-6}$.
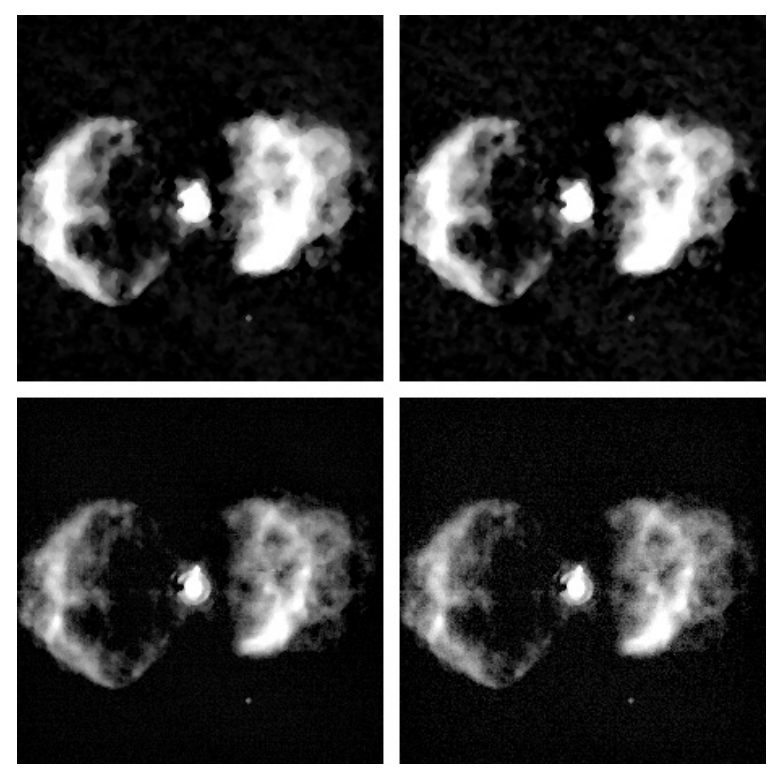

Fig. 9. Top left: result of method (B) after 100 iterations in combination with the median filtering with filter size $s=1$. Top right: result of the cg-based method after 20 iterations in combination with median filtering with size 3 resp. 1 in the last iteration. Bottom left: result of a combined reconstruction with method (B) $(\tau=0.1,100$ steps $)$ using multiple data from chopping amplitudes $(14,10),(14,0),(14,14)$, $(10,0)$, and $(7,14)$. After each iteration step the reconstructed images are reinitialized with the pointwise median. Bottom right: result of a combined reconstruction (pointwise median) with the cg-method with 20 steps using the same multiple data set.

median as described in Sect. 3.3. In Fig. 9 (bottom right) we used the cg-method (20 iterations) where again the pointwise median is calculated after each iteration.

The computation times were about $2.5 \mathrm{~s}$ using method (B) with median filtering, $0.9 \mathrm{~s}$ using the cg-method with median filtering and 2.8 or $1.6 \mathrm{~s}$ using method (B) or the cg-based method on the five different chopped data sets and calculating the median after each iteration.

\section{Summary and conclusions}

In this paper we give an overview of Fourier-based and iterative schemes for the reconstruction of images from chopped and nodded data. As an alternative to reconstruction methods documented in the literature we proposed an algorithm based on the conjugate-gradient(cg)-method which is faster converging.
Experiments show that for the "classical" reconstruction methods noise can severely affect the reconstruction process. To prevent noise enhancement we propose to combine iterative methods with median filtering techniques (robust methods from statistics). Filtering is performed in each iteration of the reconstruction process.

We produced satisfactory numerical results for three different test images with noisy chopping and nodding data. Noise artifacts are removed during reconstruction.

An alternative strategy to enhance the quality of reconstruction is to use multiple chopping amplitudes. The results of the reconstruction are combined by statistical methods after each iteration. This also provides a method robust against noise.

Since the method of multiple chopped data sets does not show any blurring effects, we propose to use this method if the required data are available. In the case of a single data set the methods with a median filter should be applied.

Acknowledgements. We thank Ulrich Kaeuffl from ESO in Garching for helpful discussions about the topic of chopping and nodding and providing the Menzel III nebula data set for testing. For running the reconstruction algorithm the computer cluster of the HPC - Konsortium Innsbruck was used. The work of F.L. is supported by the Tiroler Zukunftsstiftung. The work of O.S. and S.S. is partly supported by the Austrian Science Foundation, Projects Y-123INF, FSP Industrial Geometry 9201-N12 (subprojects 9207-N12 and 9203-N12), P15868 and the Uni Infrastructure II program.

\section{References}

Beckers, J. M. 1994, Proc. SPIE, 2198, 1432

Bertero, M., Boccacci, P., Benedetto, F. D., \& Robberto, M. 1999, Inverse Problems, 15, 345

Bertero, M., Boccacci, P., \& Robberto, M. 2000, PASP, 112, 1121

Bertero, M., Boccacci, P., Custo, A., Mol, C. D., \& Robberto, M. 2003a, A\&A, 406, 765

Bertero, M., Boccacci, P., \& Robberto, M. 2003b, Inverse Problems, 19, 1427

Chan, R., Shen, L., \& Shen, Z. 2003, Research Report, Dep. of Math., University of Hong Kong, 2003-09 (283)

Donoho, D. L., \& Johnstone, I. M. 1994, Biometrika, 81, 425

Emerson, J. P. 1994, Lecture Notes in Physics (Springer), 431, 125

Frigo, M., \& Johnson, S. G. 1998, in Proc. ICASSP, 3, 23rd International Conference on Acoustics, Speech, and Signal Processing

Hanke-Bourgeois, M. 2002, Grundlagen der Numerischen Mathematik und des wissenschaftlichen Rechnens (Teubner Verlag)

Kaeufl, U. 1995, ESO Conf. and Workshop Proc.: Calibrating and Understanding HSR and ESO Instruments, 53, 159

Kaltenbacher, B., Neubauer, A., \& Scherzer, O. 2005, Iterative Regularized Methods for Nonlinear Ill-posed problems (Springer), in press

Pestman, W. R. 1998, Mathematical Statistics (de Gruyter)

Robberto, M., Beckwith, S. V. W., Panagia, N., et al. 2005, AJ, 129, 1534

Soille, P. 2003, Morphological Image Analysis, 2nd ed. (Springer)

Sonka, M., Hlavac, V., \& Boyle, R. 1999, Image Processing, Analysis, and Machine Vision, 2nd ed. (PWS Publ.) 